\title{
Lifetime of Optical States in Transmission Electron Microscopy
}

\author{
Juri Barthel ${ }^{1,3}$ and Andreas Thust ${ }^{2,3}$
}

\author{
1. Central Facility for Electron Microscopy (GFE), RWTH Aachen University, Ahornstraße 55, 52074 \\ Aachen, Germany. \\ 2. Peter Grünberg Institute (PGI), Forschungszentrum Jülich GmbH, 52425 Jülich, Germany \\ 3. Ernst Ruska-Centre for Microscopy and Spectroscopy with Electrons (ER-C), \\ Forschungszentrum Jülich GmbH, 52425 Jülich, Germany
}

In the recent two decades the technique of high-resolution transmission electron microscopy (HRTEM) experienced an unprecedented progress through the introduction of hardware aberration correctors and by the improvement of the achievable resolution to the sub-Ångström level. With this development, also the required precision level to measure and adjust the optical properties of transmission electron microscopes has become increasingly demanding. A second important aspect of this development, which has received little attention so far, is that aberration correction at a given resolution requires also a well-defined amount of instrument stability. Therefore we investigate the qualification of a variety of high-resolution electron microscopes to maintain an aberration corrected optical state in terms of an optical lifetime.

A comprehensive statistical framework is developed for the estimation of the optical lifetime based on a simple experimental procedure [1]. The experimental procedure requires a continuous acquisition of HRTEM images of a thin amorphous object over a period of several tens of minutes in order to extract the long- and short-term temporal development of the twofold astigmatism, see Fig. 1A. The twofold astigmatism is chosen as representative indicator for the optical stability, because it is one of the most volatile aberrations, has a strong impact on the image contrast, is to a high degree free of mechanical influences, and can be monitored with high precision in real-time [2].

When aiming for aberration-free work in the sub-Ångström resolution regime, tolerable astigmatism fluctuations are often comparable or even smaller than the error of the astigmatism measurement. Therefore astigmatism data as shown in Fig. 1A are significantly contaminated by the error of the measurement procedure. We achieve an elimination of the statistical measurement error by performing our lifetime evaluation on the scale of average squared astigmatism fluctuations for a set of timespans within the recorded data series. The result of this data transformation and error correction is a characteristic curve for the temporal evolution of the twofold astigmatism, which depends on the optical stability of the instrument at the time of the investigation. The characteristic curve, which has been extracted from the astigmatism-time series shown in Fig. 1A, is displayed by the small black circles in Fig. 1B.

The optical lifetime is identified from such a characteristic curve as the timespan beyond which the average squared astigmatism fluctuation exceeds a tolerated threshold. However, this simple and direct evaluation method may fail in cases where the acquired image series is too short to allow a statistically significant statement on the average fluctuation for longer timespans. In order to overcome this limitation, a second, model-based evaluation method was developed. Within the proposed model, the astigmatism fluctuates with a random and a constant component between two exposures, realizing a combination of random walk and linear drift. When assuming a normal distribution for the random-walk component, the squared astigmatism fluctuations follow a noncentral chi-squared probability 
distribution. By applying the full statistical toolbox, the model-based approach allows us to extrapolate the observation of optical instabilities towards longer timespans (Fig 1B) and to obtain error estimates in addition. A very useful output of the statistical evaluation is a probability curve, which informs the operator about the chance to still work in an aberration-corrected state after a given timespan.

Optical stability evaluations for different high-resolution microscopes reveal surprisingly short lifetimes on the order of a few seconds up to a few minutes. The observed short lifetimes denote a critical limitation in HRTEM considering typical timespans between aberration measurement, aberration correction and the actual HRTEM imaging. Therefore further investigations and technical developments are necessary for the optical stabilization of electron microscopes with respect to their sub-Ångström qualification. Since the topic of optical stability turns out to be of similar importance as the topic of resolution itself, we recommend to include an assessment of the optical stability in acceptance tests for high-resolution microscopes operating in the discussed resolution regime.

A software program has been developed, where the evaluation schemes described in [1] have been implemented together with a convenient graphical user interface. As required input, a list of measured astigmatism values can be loaded from log files of manufacturer supplied aberration measurement routines. The program creates graphical output of each evaluation step and allows even unexperienced users to measure the optical stability of their transmission electron microscope on a routine basis. The software can be freely downloaded from a web site [3].

\section{References:}

[1] J. Barthel, A. Thust, Ultramicroscopy 134 (2013), p. 6.

[2] J. Barthel, A. Thust, Ultramicroscopy 111 (2010), p. 27.

[3] J. Barthel, http://www.er-c.org/barthel/pantarhei/(February 2014).

[4] J.B. acknowledges funding within the core facilities initiative of the German Science Foundation (DFG) under the grant number MA 1280/40-1.
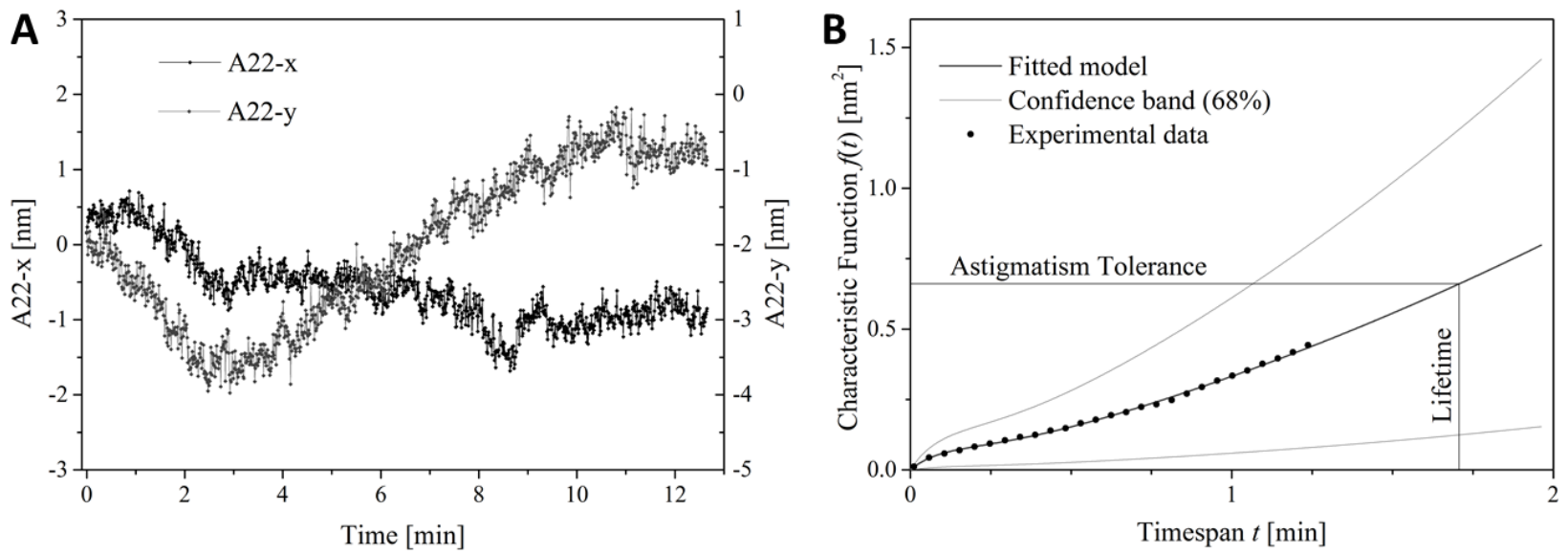

Figure 1. (A) Temporal development of the $x$ - and $y$-component of the twofold astigmatism recorded over $12 \mathrm{~min}$ from a $C_{\mathrm{S}}$-corrected high-resolution TEM operated at $300 \mathrm{kV}$ accelerating voltage. (B) Model-based evaluation of the measured astigmatism fluctuations shown on the left side, yielding a lifetime of $102 \mathrm{~s}$ for aberration free imaging with $80 \mathrm{pm}$ resolution. 